\title{
Spinal Inhibitory Neurons that Modulate Cutaneous Sensory Pathways during Locomotion in a Simple Vertebrate
}

\author{
W.-C. Li, S. R. Soffe, and Alan Roberts \\ School of Biological Sciences, University of Bristol, Bristol, BS8 1UG, United Kingdom
}

\begin{abstract}
During locomotion, reflex responses to sensory stimulation are usually modulated and may even be reversed. This is thought to be the result of phased inhibition, but the neurons responsible are usually not known. When the hatchling Xenopus tadpole swims, responses to cutaneous stimulation are modulated. This occurs because sensory pathway interneurons receive rhythmic glycinergic inhibition broadly in phase with the motor discharge on the same side of the trunk. We now describe a new whole-cell recording preparation of the Xenopus tadpole CNS. This has been used with neurobiotin injection to define the passive and firing properties of spinal ascending interneurons and their detailed anatomy. Paired recordings show that they make direct, glycinergic synapses onto spinal sensory pathway interneurons, and the site of contact can be seen anatomically.
\end{abstract}

Responses in most animals are modulated during locomotion and other motor acts by inhibition acting on central sensory pathways (Pearson, 1993). In both invertebrates and vertebrates, attention has focused on reflex modulation operating through presynaptic, $\mathrm{GABA}_{\mathrm{A}}$ inhibition of sensory axon terminals (for review, see Büschges and El Manira, 1998; Rudomin and Schmidt, 1999). This inhibition produces primary afferent depolarization, which has been studied extensively (Rudomin et al., 1998), but only in the crayfish have the inhibitory interneurons activated by the motor system been found (Kirk and Wine, 1984). The next level at which modulatory inhibition can act is through postsynaptic inhibition of sensory pathway interneurons. This occurs during fictive swimming in Xenopus tadpoles (Sillar and Roberts, 1988). In humans and other mammals, it is likely that sensory modulation also occurs in interneurons (cf. Rossignol et al., 1988; Zehr and Stein, 1999), but almost nothing is known of the mechanisms, and no interneurons controlling modulation have been characterized morphologically. Even in the lamprey, in which spinal inhibitory interneurons have been characterized, those responsible for modulation of primary afferents and central interneurons during fictive swimming have not been defined (Alford et al., 1991; El Manira et al., 1996; Buchanan, 2001).

We have explored basic mechanisms of reflex modulation in a vertebrate spinal cord using the hatchling Xenopus tadpole. This is a small and relatively simple vertebrate that can swim. At this early stage in development, the Xenopus spinal cord may contain

Received July 11, 2002; revised Sept. 12, 2002; accepted Sept. 13, 2002.

This work was supported by the Wellcome Trust and Medical Research Council. We thank Tim Colburn, Derek Dunn, Julie Hansen, Bob Porter, and Alison Walford for technical assistance and Drs. Robert Meech and Jeffrey Rohrbough for advice.

Correspondence should be addressed to Dr. Wen-Chang Li, School of Biological Sciences, University of Bristol, Woodland Road, Bristol, BS8 1UG, UK. E-mail: wenchang.li@bristol.ac.uk.

Copyright (๑) 2002 Society for Neuroscience $0270-6474 / 02 / 2210924-11 \$ 15.00 / 0$
During swimming, ascending interneurons fire rhythmically. Analysis shows that their firing is more variable and not as reliable as other interneurons, but the temporal pattern of their impulse activity is suitable to produce the main peak of gating inhibition in sensory pathway interneurons. Ascending interneurons are not excited at short latency after skin stimulation but are strongly active after repetitive skin stimulation, which evokes vigorous and slower struggling movements. We conclude that ascending interneurons are a major class of modulatory neurons producing inhibitory gating of cutaneous sensory pathways during swimming and struggling.

Key words: locomotion; CPG; reflex reversal; spinal cord; glycine; Xenopus

as few as eight classes of neuron, each with distinctive anatomy (Roberts and Clarke, 1982; Roberts, 2000). The functions of some of these neurons have been defined by single neuron recording and dye marking. Skin touch can initiate or speed up swimming by exciting skin sensory Rohon-Beard neurons (Clarke et al., 1984). These excite sensory pathway interneurons, which project across the cord to excite motoneurons on the opposite side (see Fig. 1A) (Roberts and Sillar, 1990). During swimming, the sensory pathway interneurons receive glycinergic inhibition broadly in phase with the motor discharge on the same side of the trunk (Sillar and Roberts, 1992a). This inhibition gates the sensory pathways from skin touch, so excitation can strengthen ongoing trunk contractions on the opposite side (Sillar and Roberts, 1988, 1992a,b). It was proposed that this inhibition was mediated by the ipsilateral projections of reciprocal inhibitory interneurons whose principal projection was to the antagonistic motor system on the opposite side (Dale, 1985; Dale et al., 1990; Sillar and Roberts, 1992). However, doubt has arisen about this proposal because a large sample of these interneurons has now been examined, and very few have ipsilateral projections (Yoshida et al., 1998).

Single and paired whole-cell recordings from spinal interneurons have now been used in the semi-intact immobilized Xenopus tadpole to search for inhibitory interneurons that could produce gating of cutaneous sensory pathway interneurons. We describe the properties and activity patterns of spinal ascending interneurons and show that they are active during swimming and produce suitable glycinergic inhibition of sensory pathway interneurons.

\section{MATERIALS AND METHODS}

Whole-cell recordings. Xenopus tadpoles at stage 37-38 (Nieuwkoop and Faber, 1956) (see Fig. 1B) were anesthetized with $0.1 \%$ 3-aminobenzoic acid ester (Sigma, Poole, UK) for $20 \mathrm{sec}$ and then pinned onto a small rotatable Sylgard (Dow Corning, Midland, MI) stage in a bath of saline 
(concentrations in mM: $115 \mathrm{NaCl}, 3 \mathrm{KCl}, 2 \mathrm{CaCl}_{2}, 2.4 \mathrm{NaHCO}_{3}, 1$ $\mathrm{MgCl}_{2}$, and $10 \mathrm{HEPES}$, adjusted with $5 \mathrm{M} \mathrm{NaOH}$ to $\mathrm{pH} 7.4$ ). In many paired recording experiments, $1 \mathrm{mM} \mathrm{MgCl}_{2}$ was replaced by $1 \mathrm{mM} \mathrm{CaCl}_{2}$. The dorsal fin was cut open with a finely etched tungsten needle, and the tadpole was transferred to $10 \mu \mathrm{M} \alpha$-bungarotoxin in saline for $20 \mathrm{~min}$. After immobilization, it was repinned on the Sylgard stage. The dorsal trunk skin and muscles over the right side of the spinal cord were removed. Then a dorsal cut was made along the midline of the spinal cord to open the neurocoel in the exposed area (see Fig. 1C). Loose tissue and cells were removed. To expose more ventral neurons, some ependymal cells on the right side of the neurocoel were also removed. Finally, the whole of the ventral part of the trunk (largely yolk sac) was removed to allow better illumination. The animal was then moved to a $700 \mu \mathrm{l}$ recording chamber and repinned on a small rotatable Sylgard stage (see Fig. $1 B$ ). The bottom of the recording chamber was a piece of replaceable coverslip, and there was a gap in the Sylgard stage beneath the tadpole to allow bright-field illumination from below on an upright Nikon E600FN microscope (Tokyo, Japan). The animal was tilted to an angle that allowed the exposed cell bodies on the left and right sides of the cord to be seen under the microscope (see Fig. $1 C$ ). Saline in the chamber was circulated at $\sim 2 \mathrm{ml} / \mathrm{min}$. Antagonists were added to another $100 \mu \mathrm{l}$ chamber upstream of the recording chamber. Bicuculline and strychnine were from Sigma.

Patch pipettes were filled with $0.1 \%$ neurobiotin in intracellular solution (concentrations in mM: $100 \mathrm{~K}$-gluconate, $2 \mathrm{MgCl}_{2}, 10 \mathrm{EGTA}, 10$ HEPES, $3 \mathrm{Na}_{2} \mathrm{ATP}$, and $0.5 \mathrm{NaGTP}$ adjusted to $\mathrm{pH} 7.3$ with $\mathrm{KOH}$ ) and had resistances of 5-10 M $\mathrm{M}$. Stimulating suction electrodes were placed on head and tail skin to start fictive swimming or struggling activity. Another suction electrode was placed usually on the 10th intermyotome cleft to record ventral root activity. Patch pipettes were motor driven by two MX 763OR manipulators from SD Instruments. Under a $40 \times$ water immersion lens, patch pipettes were advanced to contact exposed interneuron somata. Positive pressure $\left(5-20 \mathrm{~cm} \mathrm{H}_{2} \mathrm{O}\right)$ was always applied to the pipette solution before trying to get a seal. In some neurons, extracellular recordings were made in cell-attached mode before trying to obtain whole-cell mode or after the positive pressure was released. Extracellular or intracellular signals were recorded with Axoclamp 2B in conventional bridge mode and sometimes amplified 5 or $50 \times$. Data were acquired with Signal software through a CED 1401 Plus with sampling rate of $10 \mathrm{kHz}$. Stimuli to the skin were controlled using the CED 1401 Plus configured by Signal and given via an optically coupled isolator. Off-line analyses were made with Minitab and Excel.

A total of 81 ascending interneurons (aINs) with somata between the third and eighth segments were recorded in this study. They were identified using anatomical features described in Li et al. (2001) and physiological features established in this study. In 14 cases, neuronal somata and dendrites broke when the electrode was withdrawn after recording, but the neurons could still be identified by their unique axons and physiology. The somata of the remaining 64 aINs were located between 0.88 and $1.97 \mathrm{~mm}$ from $\mathrm{mid} /$ hind brain border. For analysis of neuron properties, 59 with stable resting membrane potentials of -50 $\mathrm{mV}$ or more were used.

Neuron labeling. Once physiological testing was completed, trains of positive current pulses (10-100 pA, $500 \mathrm{msec}$ duration) were applied for 2-5 min to label recorded neurons. The tadpoles were then left for $30 \mathrm{~min}$ to allow the neurobiotin to spread. Animals were fixed in $2 \%$ glutaraldehyde in $0.1 \mathrm{M}$ phosphate buffer, $\mathrm{pH} 7.2$, overnight in the refrigerator $\left(\sim 4^{\circ} \mathrm{C}\right)$. After they were rinsed with $0.1 \mathrm{M}$ PBS $(120 \mathrm{~mm} \mathrm{NaCl}$ in $0.1 \mathrm{M}$ phosphate buffer, $\mathrm{pH} 7.2$ ), the animals were (1) washed in two changes of $1 \%$ Triton X-100 in PBS for 15 min with agitation, (2) incubated in a 1:300 dilution of extravidin peroxidase conjugate (Sigma) in PBS containing $0.5 \%$ Triton X-100 for 2-3 hr with agitation, (3) washed again in at least four changes of PBS, (4) presoaked in $0.08 \%$ diaminobenzidine in PBS (DAB solution) for $5 \mathrm{~min}$, (5) moved to a second container with $0.075 \%$ hydrogen peroxide in DAB solution for $5 \mathrm{~min}$, and (6) washed in running tap water. The brain and spinal cord were then removed together with the notochord and some ventral muscles, dehydrated, cleared in methyl benzoate and xylene, and mounted whole, between two coverslips using Depex.

Neurons were observed using a $100 \times$ oil immersion lens and traced to record neuron features at $1 \mu \mathrm{m}=1 \mathrm{~mm}$ using a drawing tube. Axonal projections were traced at 5 or $10 \mu \mathrm{m}=1 \mathrm{~mm}$. Measurements were made from the scale drawings, and neuron positions are referenced to postotic myotome segment numbers. To compensate for shrinkage during dehydration, all measurements in this study have been corrected by multiply- ing by 1.28 (Li et al., 2001). All means are given with their SD unless stated otherwise.

\section{RESULTS}

Sharp electrode recordings have shown that spinal sensory pathway dorsolateral commissural interneurons (dlcs) are modulated by IPSPs during fictive swimming (Fig. 1A) (Roberts and Sillar, 1990). Most of these IPSPs are loosely in-phase with ipsilateral motor root activity, although some can also occur loosely in-phase with contralateral motor root activity (we refer to these phases as "on-cycle" and "mid-cycle," respectively), and, as swimming slows, cycles can occur without any IPSPs (Sillar and Roberts, 1992a). The inhibitory interneurons producing these IPSPs must be active during swimming. Neurons in a given longitudinal position on one side of the spinal cord are active at the same time and tend to fire a single action potential during each cycle (Soffe and Roberts, 1982; Soffe et al., 1984). The inhibitory interneurons producing on-cycle IPSPs would therefore need to have ipsilateral axonal projections. Anatomical results have shown that aINs have ipsilateral axons, which often run in positions where they could contact the dendrites of dlcs (Li et al., 2001). We therefore analyzed whole-cell recordings from these interneurons.

\section{Recording the activity of alNs during swimming}

As the study proceeded, we were able to recognize probable aINs by their characteristic "irregular" pattern of spiking during swimming, activity during struggling, and responses to current (see below). However, identification as an aIN was based on anatomical features determined after fixation and processing to reveal the injected neurobiotin. All aINs in the present study had the following features defined in our previous study of spinal interneuron classes (Li et al., 2001): (1) unipolar soma, (2) quite extensive and mainly ventral dendrites, and (3) ascending ipsilateral axon that branches to give an ipsilateral descending axon (Figs. $2 A, C, 4 A, D, F, 12 A$ ).

The recordings in Figure 2 show the two typical activity patterns of aINs during swimming. aINs are depolarized and receive oncycle excitation, which can lead to firing. A minority of aINs are active on many cycles during swimming (Fig. $2 A, B$ ), but most fire only a few spikes at the start of each swimming episode and are then usually silent (Fig. 2C,D). A few aINs fall between these extremes. Some aINs receive clear mid-cycle inhibition (Fig. 2D), whereas in many, the inhibition is less clear (Fig. 2B). The detailed firing patterns of these interneurons are considered below.

Because we had not used whole-cell recording previously in the Xenopus tadpole spinal cord, an important step was to evaluate the recording technique. An electrode was advanced until it contacted the exposed surface of an interneuron soma seen protruding from the surface of the spinal cord. Fictive swimming was evoked by a $1 \mathrm{msec}$ current pulse to either the head or tail skin, which is equivalent to a brief touch to the skin (Clarke et al., 1984). Once in contact with the soma and after positive pressure was released, the spike activity of the aIN could be recorded in two ways: (1) extracellular, before the electrode sealed onto the cell surface, and (2) cell-attached, after the electrode sealed onto the soma membrane. Recordings using both of these methods are shown in Figure 2, where they are compared with whole-cell recordings made after application of suction to break through the soma membrane. The two examples show that very similar spike activity is recorded during swimming both before and after going whole-cell. The similarity here and in 10 other aINs in which the comparison was made gives us confidence that whole-cell recording is not changing the activity of the neurons. 

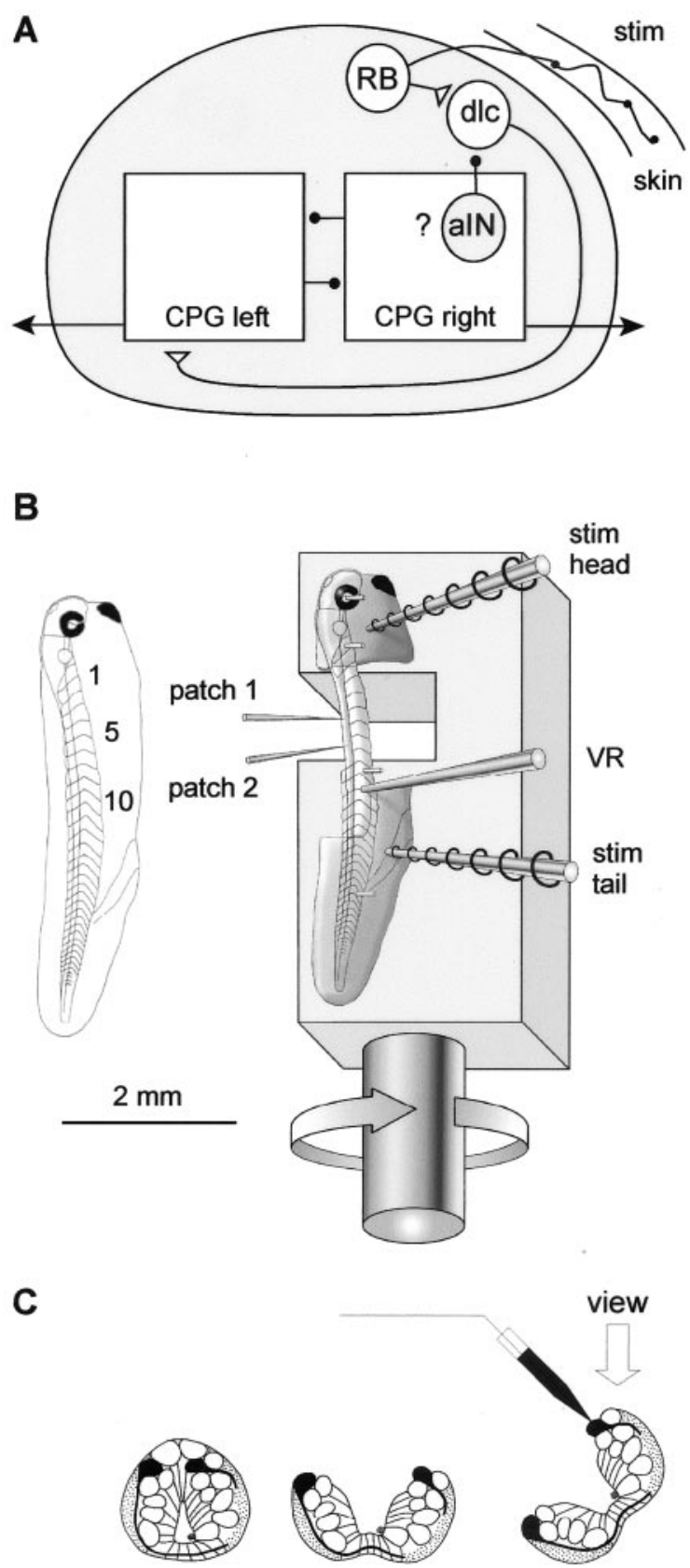

Figure 1. Diagram of the spinal cord pathways under study and the preparation of the Xenopus tadpole for whole-cell recording. $A$, Diagrammatic section through the spinal cord showing the sensory pathway from the skin to the spinal CPG for swimming. $R B$, Rohon-Beard neurons; $d l c$, sensory pathway interneurons; $a I N$, possible inhibitory gating ascending interneurons. Open triangles, Excitatory synapses; closed circles, inhibitory. $B$, Diagram of whole stage 37-38 tadpole with some muscle segment numbers (left) and after dissection (right) when pinned to the rotatable rubber block in the recording bath (bath not shown). stim head, stim tail, Stimulating electrodes; VR, ventral root electrode; patch 1, patch 2, whole-cell patch electrodes. $C$, Diagram of the tadpole spinal cord in transverse section (left), opened dorsally (middle) and rotated (right) so that exposed neuronal somata (black) can be seen from above; patch electrodes were placed on selected somata.

\section{Properties of alNs}

Having established that the whole-cell recording technique was not significantly changing recorded activity during swimming, we used current injection to measure the basic response characteris- tics of aINs. They had resting membrane potentials of $-58.1 \pm 4$ $\mathrm{mV}(n=59)$, and responses to current injection were generally linear around the resting membrane potential (Fig. 3A) $(n=10)$. Their input resistance (measured from the slope of the currentvoltage relationship or using a single hyperpolarizing current pulse) ranged from 270 to $2467 \mathrm{M} \Omega(1140 \pm 540 \mathrm{M} \Omega ; n=28)$. Input resistance was not correlated with resting membrane potential. The impulse firing threshold for 27 aINs was measured as the peak level of EPSPs that were not big enough to evoke spikes during swimming or struggling activity. The threshold measured in this way was $-23.7 \pm 4.1 \mathrm{mV}$. This is equivalent to $34 \mathrm{mV}$ depolarized from the mean resting potential. Firing thresholds to injected depolarizing current steps were not investigated in detail, but measurements from seven aINs gave results similar to those using EPSPs, with the largest subthreshold responses being $27.9 \pm 5.3 \mathrm{mV}$ depolarized from the resting potential $(n=7)$. All aINs, except for the one with the lowest input resistance, showed nonadaptive firing when positive current injection was above threshold (Fig. 3B). The firing frequencies were from 4 to $142 \mathrm{~Hz}$ and increased broadly logarithmically as current intensity increased (Fig. $3 B)(n=8)$. At low levels of injected current $(<50$ $\mathrm{pA}$ ), neurons with higher input resistances fired at higher frequencies to the same current level, but at higher current levels there was no significant correlation between input resistance and firing frequency.

When compared with previous sharp electrode recordings from other spinal neurons (Soffe, 1990), aINs have less negative resting potentials, less negative impulse firing threshold, multiple, overshooting impulses to current injection, and a wide range of higher input resistances. Despite the wide range of input resistances, all aINs have rather consistent firing properties.

\section{Paired recordings from alNs and dlcs}

To establish whether aINs made inhibitory synaptic contacts onto dlcs, we made simultaneous recordings from 17 pairs of these two types of interneurons on the right side of the spinal cord. During the experiments, dlcs were recognized by their short latency excitation after ipsilateral trunk skin stimulation and mainly oncycle IPSPs during swimming. Candidate aINs were recognized by their irregular firing activity during fictive swimming. In all cases, neuron identities were established neuroanatomically after the experiment (Fig. 4A,D,F). The criteria for identification of dlcs (Roberts and Sillar, 1990; Li et al., 2001) were slightly adapted because the most dorsal part of the spinal cord is removed when the cord is opened along its dorsal midline (Fig. 1C). All dlcs had the following features: multipolar soma at the dorsal edge of the opened spinal cord, dendrites mainly arising from the soma and lying in the dorsal region of spinal cord, and an axon that runs ventrally where it crosses to the opposite side to branch and then ascend and descend.

When current was injected to induce impulse firing in the recorded neurons, the only interactions found were inhibitory and from the aIN to dlcs $<0.46 \mathrm{~mm}$ away either rostral or caudal. In 11 cases, there was an inhibitory interaction during which, on at least five trials, an aIN spike produced an IPSP in the dlc. Examples in which aIN spikes produce IPSPs in dlcs are shown in Figures 4 and 5.

IPSP latencies (measured from the peak of the presynaptic spike to $10 \%$ of the peak amplitude on the IPSP) were short, in the range $1.1-3 \mathrm{msec}(2 \pm 0.6, n=9$ neuron pairs; Fig. $4 B)$. They also had low SDs for each cell pair (range $0.12-0.44 \mathrm{msec}$ ). We therefore conclude that they were monosynaptic. This conclusion 

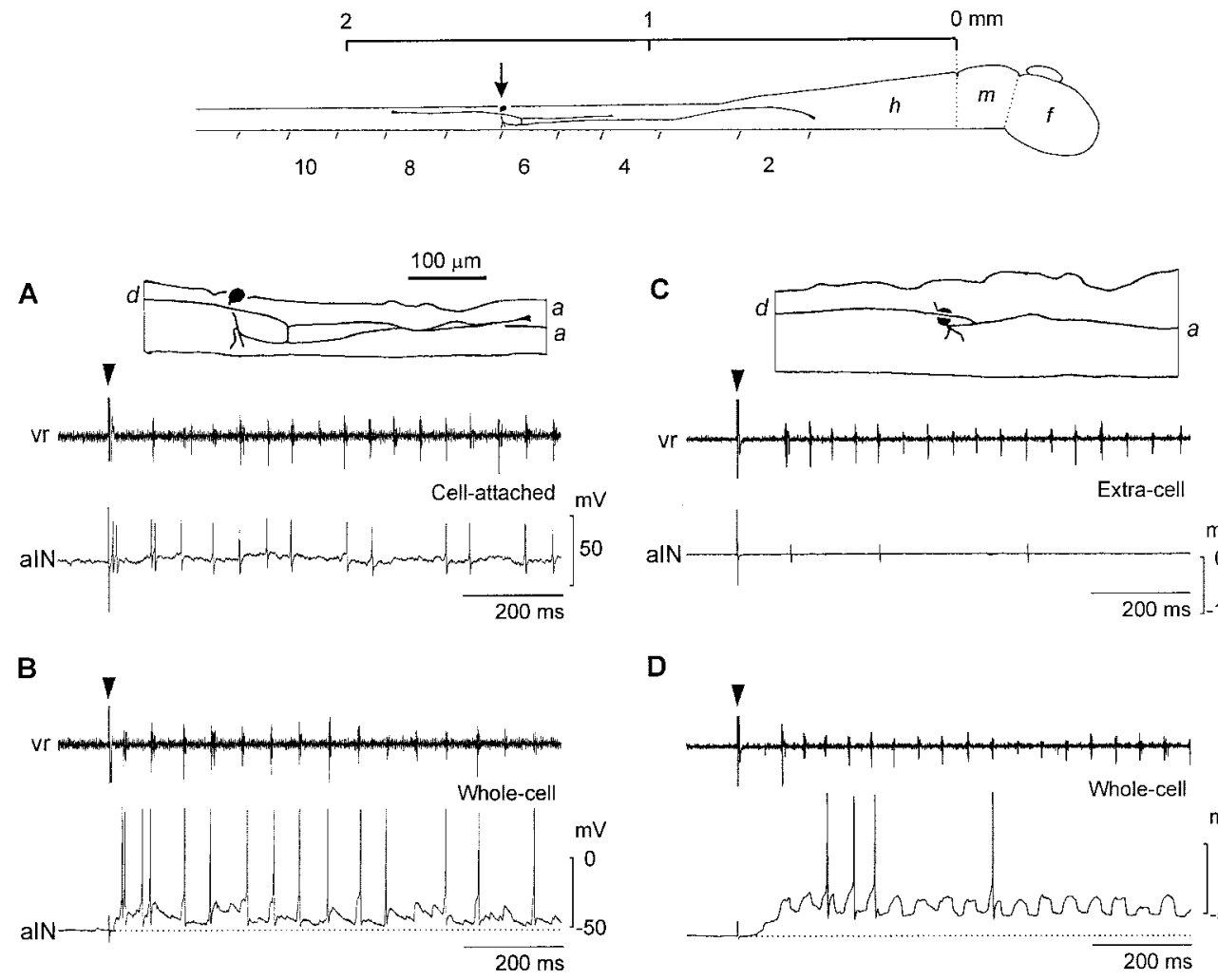

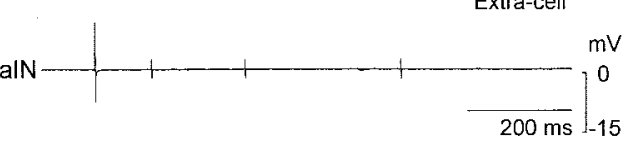

D

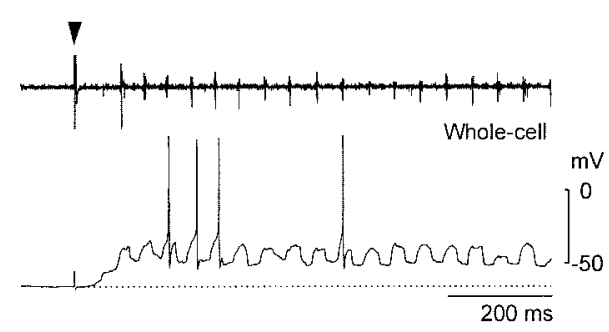

Figure 2. Examples of aIN anatomy and activity during swimming. Diagram at top shows lateral view of CNS with brain to the right with forebrain $(f)$, midbrain $(m)$, and hindbrain $(h)$. Arrow marks aIN in spinal cord $\sim 1.5 \mathrm{~mm}$ from midbrain, with two ascending and one descending axons. Numbers indicate muscle segments. $A, C$, Scale diagrams of the anatomy of two right-side aINs (somata black) in whole-mount spinal cords; rostral is to the right. Each aIN has one or two ascending axons $(a)$ that branch to form a descending axon $(d)$. In each of the records, the top trace shows swimming activity in a ventral root $(v r)$ after a skin stimulus (arrowhead), and the bottom trace shows the activity of the aIN. $A$, aIN at the seventh segment seen in lateral view (also shown in diagram at top). It fired frequently during swimming in cellattached mode and also after whole-cell recording was established $(B) . C$, aIN at the fourth segment seen in oblique ventral view. Extracellular recording shows that it was active only near the start of swimming. D, Activity was similar during whole-cell recording. was endorsed by the observation of close contact between the aIN axon and dlc soma or dendrites in eight of these recorded pairs (Fig. $4 A, F$, arrows). Because axon projections and the probable sites of synaptic contact could often be identified from the neurobiotin fills of presynaptic and postsynaptic neurons (Fig. 4A, F), conduction distances could be measured directly. These ranged from 0.134 to $0.518 \mathrm{~mm}$. Latency tended to increase with distance $(p=0.057)$; the $y$-intercept of the regression line suggested a synaptic delay of $\sim 1 \mathrm{msec}$, and the slope gave a conduction velocity of $\sim 0.33 \mathrm{~m} / \mathrm{sec}$ (cf. Clarke et al., 1984). IPSPs increased in amplitude with depolarization and reversed with hyperpolarization (Fig. 4C). Their rise times (measured between 10 and $90 \%$ peak amplitude) were in the range $2.2-6.4 \mathrm{msec}$ (4.67 \pm $1.43 ; n=9$ ), and durations (measured at $50 \%$ peak amplitude) ranged from 15.1 to $43.8 \mathrm{msec}(29.25 \pm 9.95)$ (Figs. $4 B, C, G, 5)$. Rise time and duration were not significantly correlated. At both slow and rapid repetition rates, IPSP amplitude was variable in all cases, and there were frequent failures (Fig. $4 E$ ). This variability was also clear during swimming activity (Fig. $4 H, I$ ), but it was not examined in detail.

In three cases, it was possible to estimate the peak conductance of IPSPs in dlcs from aINs by comparing the slopes of $I / V$ responses to a series of injected current steps measured before, and at the peak of, each IPSP (cf. Soffe et al., 2001). Conductances measured in this way (which ignores the membrane time constant and therefore gives an underestimate) were $0.41,1.63$, and $1.78 \mathrm{nS}$. The IPSP reversal potentials for the same three examples were $-62,-51$, and $-51 \mathrm{mV}$, respectively. These reversal potentials were close to the resting potential of the neurons.

Previous studies on the pharmacology of the dlc IPSPs during swimming had shown that they were blocked by the glycine antagonist strychnine but unaffected by the GABA antagonists bicuculline and curare (Sillar and Roberts, 1992a; Soffe 1993). In the present study, IPSPs evoked in dlcs by aIN impulses were blocked by strychnine ( 1 or $5 \mu \mathrm{M} ; n=8$ ) but not bicuculline (10 $\mu \mathrm{M} ; n=2$ ) (Fig. 5).

\section{Timing of alN activity during swimming and comparison with dlc IPSPs}

If aINs are the neurons that produce the modulating inhibition in dlcs, then their pattern of firing during swimming should correlate with the pattern of IPSPs seen in dlcs. We therefore examined these two patterns in detail.

The pattern of spike activity in aINs was revealed by displaying traces of swimming cycles after the cycle length was normalized and by spike phase plots (Fig. 6A,B). Cycle timing was determined by the onset of consecutive ventral root bursts, and cycles were normalized to phase values between 0 and 1 . However, there is a rostrocaudal delay in ventral root activity during swimming. The timing of ventral root activity was therefore corrected for longitudinal distance from the recorded neuron by assuming a delay of $3.5 \mathrm{msec} / \mathrm{mm}$ (Tunstall and Roberts, 1991), before normalizing each cycle. aINs fired broadly on-cycle during swimming but later than the start of ipsilateral motor root bursts and, therefore, the motoneurons that produce them (Fig. 6A-C). Spikes in aINs were also not closely synchronized. They could occur anywhere during the cycle, although most occurred during the first half (phase $0-0.5$ ). aINs could also fire more than one spike per cycle, particularly (although not exclusively) near the start of a swimming episode (see below).

For comparison with aINs, we also examined the firing pattern of a sample of five of the best-known class of premotor interneuron in the spinal cord, the reciprocal commissural inhibitory interneurons (cINs) (Soffe et al., 1984; Dale, 1985). It was originally proposed that ipsilateral connections from 
A
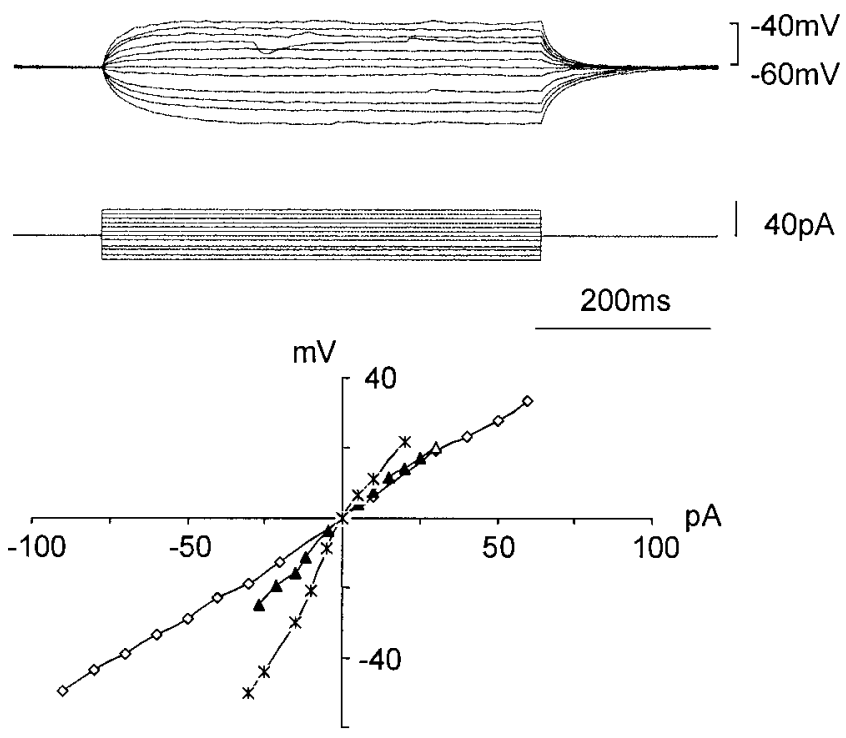

B
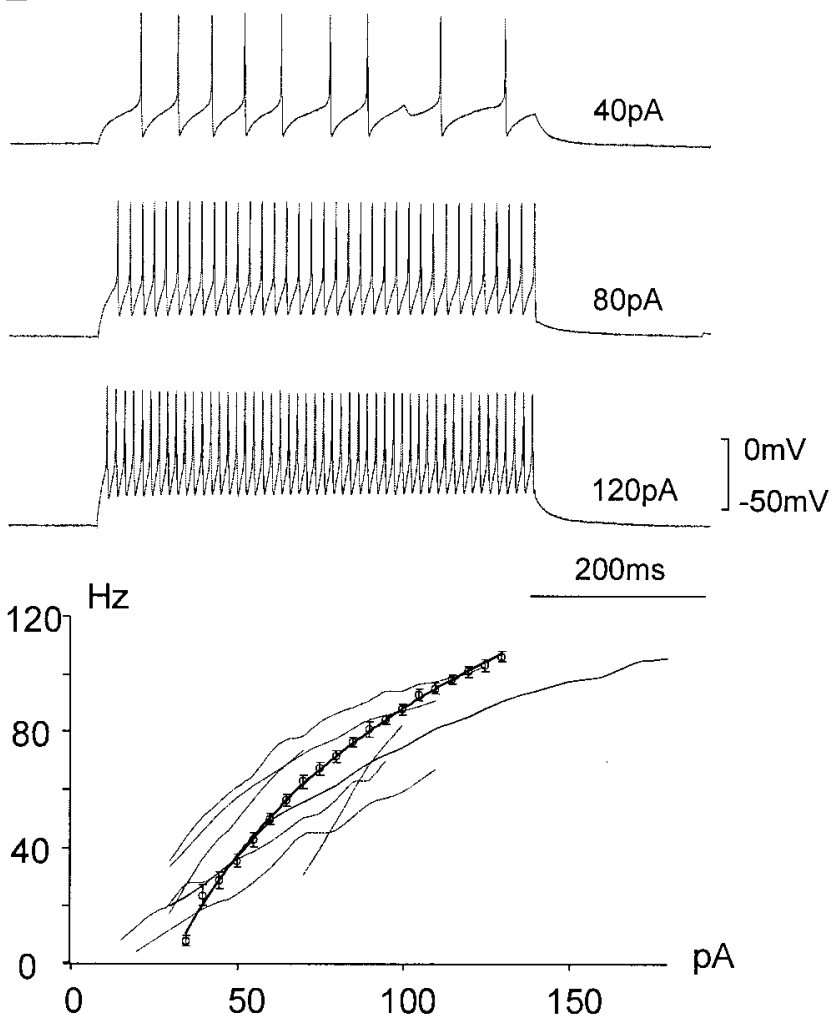

Figure 3. Responses of aINs to injected current. $A$, Responses to depolarizing and hyperpolarizing current in a typical aIN with $R_{\text {in }}$ of $938 \mathrm{M} \Omega$. Plot shows current-voltage relationship for the records shown $(\boldsymbol{\Delta})$ and two other aINs with higher (crosses, $R_{\text {in }}=1770 \mathrm{M} \Omega$ ) and lower $\left(\diamond, R_{\text {in }}=\right.$ $562 \mathrm{M} \Omega) R_{\text {in }}$. $B$, Responses to three levels of suprathreshold current injection to show nonadapting, repetitive firing. Plot shows firing frequency as a function of injected current for this neuron (heavy line, $\pm \mathrm{SD}$; regression $R^{2}=0.9984$ ) and seven other neurons (fine lines).

some of these neurons were the source of IPSPs in dlcs (Dale, 1985). In contrast to aINs, cINs fired only once per swimming cycle, and their firing was highly synchronized, with peak firing occurring just before each ipsilateral ventral root burst (Fig.
$6 D)$, therefore at the same time as motoneurons. The only cIN recorded in this survey with an ipsilateral axon in addition to its conventional contralateral axon shared the same firing pattern as the other cINs. The short duration of ventral root bursts during swimming (Figs. 2, 6A) shows that motoneurons, like cINs, have a very synchronized pattern of firing, which is distinct from that of aINs.

The phase of onset of IPSPs was then measured for 12 dlcs in eight animals. Most IPSPs were broadly on-cycle, but they were not closely synchronized, and IPSPs could occur anywhere during the cycle (Fig. 7). As well as the main on-cycle peak, some dlcs showed a second broad peak of IPSP phases starting after midcycle (Fig. 7B).

As a population, the firing phases of aINs were closely correlated with the distribution of IPSP phases in dlcs (Pearson correlation $0.848 ; p<0.001$ ) (Fig. $8 A$ ). To allow for conduction times and synaptic delays between spikes and IPSPs (see above), correlations were tested after correcting the phase of spikes by 0.05 (equivalent to $\sim 3 \mathrm{msec}$ ). In contrast, the firing pattern of ipsilateral cINs showed no significant correlation with the timing of dlc IPSPs (Pearson correlation 0.056; $p=0.813$ ). Their spikes occurred relatively too soon (just before on-cycle) and with too narrow a phase distribution (Fig. $8 A$, dashed line). The firing pattern of these cINs was also inappropriate to explain the second, smaller peak of IPSPs occurring in some dlcs after mid-cycle. It is probable that these later IPSPs result from spikes in contralateral neurons with commissural axons, because we have not encountered any ipsilateral neurons that fire reliably at around mid-cycle. However, the distribution of these later IPSPs was again too broad and too late in the cycle to match the firing of contralateral cINs (Fig. 8, compare $A, B$ ). The origin of the later IPSPs in dlcs therefore remains unclear. In contrast, the timing of mid-cycle IPSPs in aINs correlated strongly with the firing of cINs after the timing of the latter had been advanced by half a cycle (phase $=0.5$ ) to imitate the timing of their contralateral homologs (Pearson correlation 0.938; $p<0.001$ ) (Fig. 8B). We conclude that mid-cycle IPSPs in aINs during swimming come from contralateral cINs.

\section{Activity of alNs during swimming episodes}

During episodes of swimming, aINs were mainly active at the start and could fire multiply on each cycle (Figs. 1, 6A, 9B). Although a few aINs continued firing throughout episodes (Fig. $9 A$ ), spiking in most aINs became sporadic and generally decreased with time (Figs. 1C,D, 9B). The early firing of most aINs produced a strong peak of spikes at the start of each episode (Fig. $9 C)$. However, when a sample of aINs was considered as a group $(n=17)$, the more reliable firing aINs, although fewer in number ( 2 of 17 fired on $>60 \%$ of cycles), made a significant contribution during the remainder of each episode.

The pattern of synaptic drive to alNs during swimming

The pattern of synaptic drive to aINs was broadly similar to that described for motoneurons and premotor interneurons on the basis of sharp microelectrode recordings (Soffe and Roberts, 1982; Soffe et al., 1984; Roberts et al., 1997, 1998). However, whole-cell recordings showed that the pattern of synaptic drive in aINs was more variable. On-cycle excitation was usually clear, and in some cases it summed to produce a steady depolarization (Fig. 2B,D). Mid-cycle IPSPs (in time with motor activity on the opposite side) were frequently hard to distinguish unless the membrane potential of the neuron was manipulated to enhance 


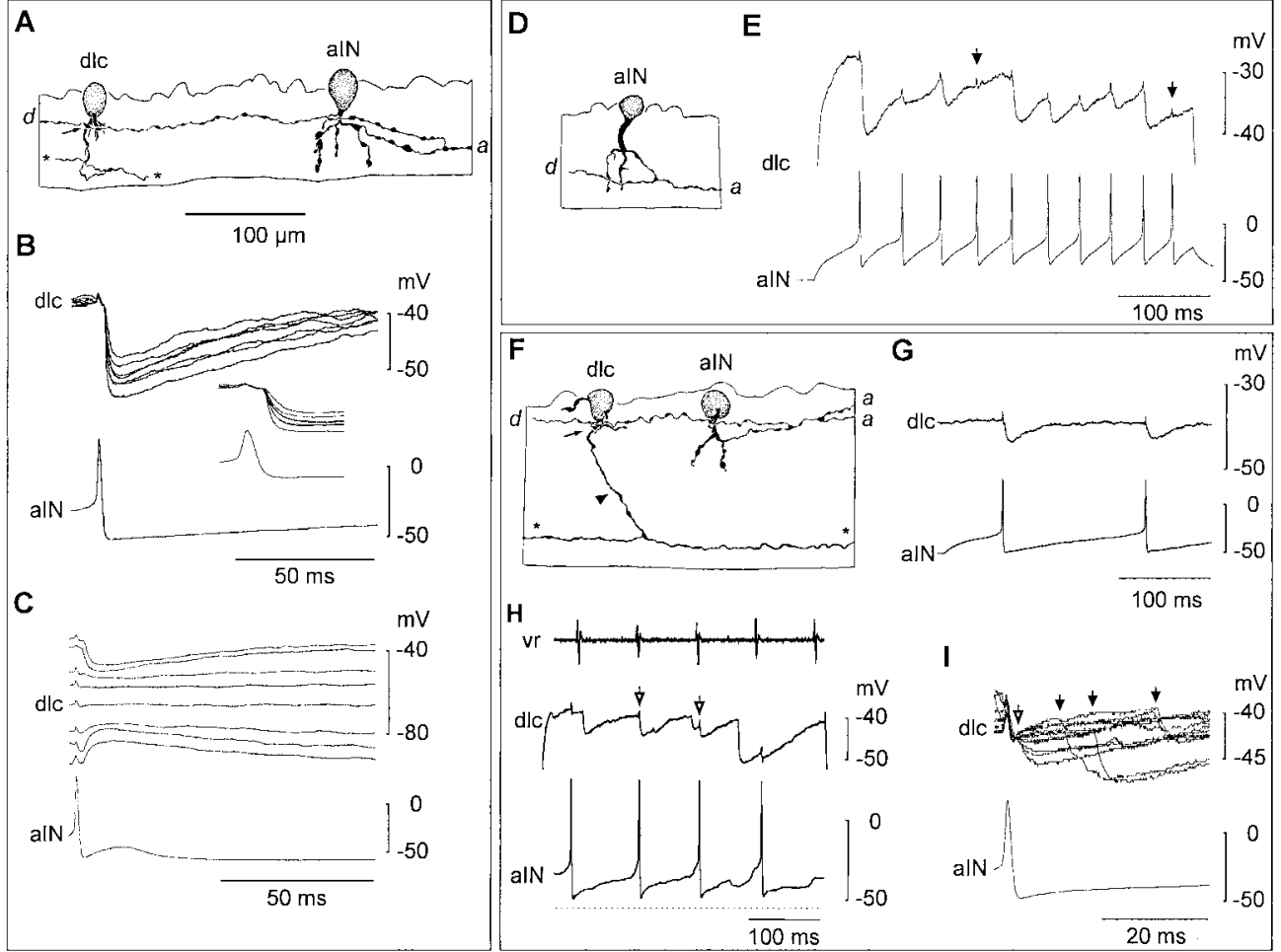

Figure 4. Examples of three paired recordings (each in a box) show that aINs inhibit dlcs. $A, D, F$, Scale drawings of three examples in which an aIN inhibited a dlc. In each case, rostral is to the right, the exposed edge of the dorsal spinal cord is at the top, somata are stippled, $a$ is the ascending axon of the aIN, and $d$ is the descending ipsilateral axon of the aIN; asterisks mark contralateral axons of dlcs, and arrow indicates area of synaptic contact. $A, D$, Viewed from the right side. Scale in $A$ applies also to $D$ and $F$. $A$, aIN (fourth segment) contacting dlc (fifth segment) via its descending axon. $B$, Currentinduced spikes in the aIN cause a small cross-talk artifact in the dlc followed by IPSPs at short latency. The dlc was depolarized to increase the IPSP amplitude. Inset at expanded time scale (trace duration $12.5 \mathrm{msec}$ ) shows the low variance in IPSP latency. $C$, Spikes evoked in the aIN by current evoke IPSPs in the dlc that increase in amplitude with depolarizing current and reverse with hyperpolarizing current. $D$, aIN (eighth segment), which contacted a dlc 0.46 $\mathrm{mm}$ more rostral (sixth segment). E, Current injection into the aIN evokes a train of spikes. The first evokes a large IPSP in the dlc, after which the IPSPs are variable in amplitude or fail (arrows). F, aIN (fourth segment) and dlc (fifth segment) seen in oblique ventral view; arrowhead marks the point at which the ventral commissural axon of the dlc crosses the midline. $G$, Current-evoked aIN spikes lead to IPSPs in the dlc. $H$, During swimming, shown by an ipsilateral ventral root record ( $v r$ ), some aIN spikes appear to correlate with a dlc IPSP (open arrows), whereas others do not. I, When traces are aligned to aIN spikes during swimming, some IPSPs are correlated with the spikes (open arrow), whereas others (solid arrows) are not.
A

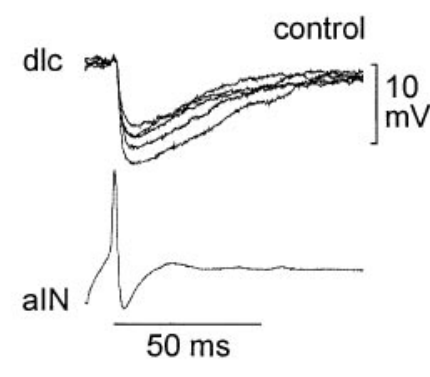

B

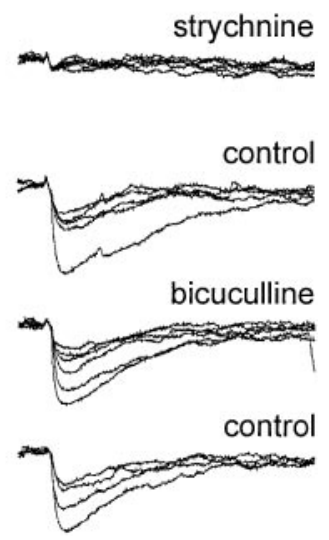

Figure 5. Pharmacology of dlc IPSPs evoked by aINs. $A$, dlc IPSPs (revealed by depolarizing the dlc to approximately $-27 \mathrm{mV}$ using current injection) in traces aligned to the aIN spike showing their short and constant latency. $B$, These IPSPs are completely blocked by bath application of the glycine antagonist strychnine $(5 \mu \mathrm{M}, 75 \mathrm{sec})$, recover in wash (control), and appear not to be affected by application of bicuculline (10 $\mu \mathrm{M}, 85 \mathrm{sec})$ and subsequent wash (control).

them (by depolarization) or reverse them (by hyperpolarization) (Fig. 10A). The occurrence of mid-cycle IPSPs was rather irregular; they were present on most cycles in some aINs (Figs. 2D, $10 A$ ) but on only a few, if any, cycles in others (Fig. 10B). The on-cycle excitation was also obviously variable in shape; it was sometimes small in amplitude, and the initial part often lacked the clearly defined peak seen in other spinal neurons (Fig. 10A,B). Despite this, injection of depolarizing current readily produced firing in aINs that otherwise gave few, if any, spikes during swimming (Fig. 10A,B) and increased the number of spikes per cycle in those already firing.

To quantify the cycle-by-cycle variability in the pattern of synaptic drive to aINs during swimming, deviation from the mean membrane potential trajectory was examined over 20 cycles in five neurons. Cycles were first normalized for length, and cycles by irregularly occurring spikes. Figure 10 shows the mean membrane potential trajectory and its SD for two aINs. In one, the on-cycle excitation rises to a distinct peak, but the mid-cycle IPSP is unclear (Fig. 10C), whereas in the other, excitation has a broadly rounded peak, and the IPSP is clear (Fig. 10D). We measured the SD during the excitation just before mid-cycle and during inhibition just after mid-cycle (Fig. 10C,D, cycle phases of 0.4 and 0.6$)$. The values were $3.42 \pm 1.57 \mathrm{mV}$ for excitation, $2.81 \pm 1.76 \mathrm{mV}$ for inhibition $(n=5 \mathrm{aINs}), 22$ to $50 \%$ of the mean peak-to-peak amplitude of the synaptic drive $(10 \pm 2.1$ $\mathrm{mV})$. This variability of the synaptic drive in aINs was significantly higher than for cINs at both phases ( $p=0.018$ and 0.039 , respectively; $n=5$ of each neuron class). The SDs in cINs were $1.27 \pm 0.42 \mathrm{mV}$ for excitation and $0.83 \pm 0.36 \mathrm{mV}$ for inhibition, corresponding to a maximum of only $14 \%$ of the mean peak-topeak amplitude. The significance of the variability in synaptic drive to aINs is unclear, but it seems likely that it will be a factor in producing the rather variable pattern of firing seen in these neurons during swimming.

\section{Other possible roles for alNs}

Because aINs produce glycinergic IPSPs in ipsilateral dlcs during swimming, it was possible that some of them could also be without spikes were selected to avoid distortion of measurements 
A

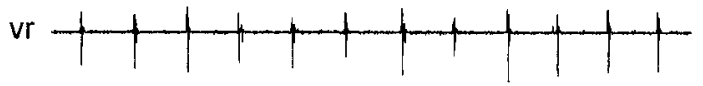

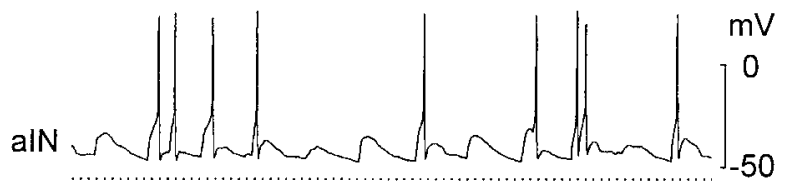

B

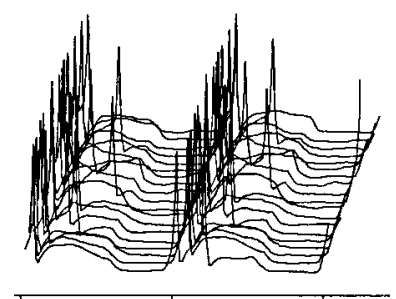

C

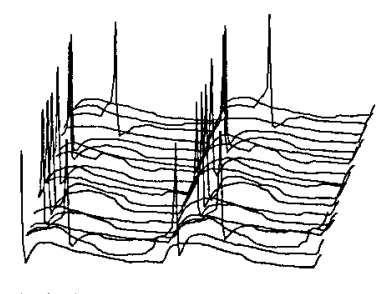

D

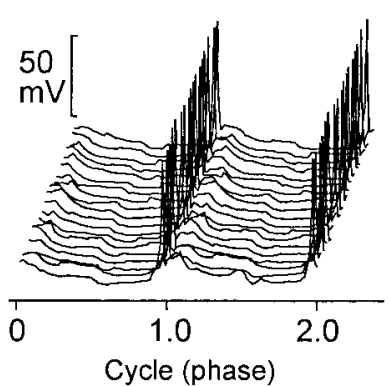

$\overline{100 \mathrm{~ms}}$
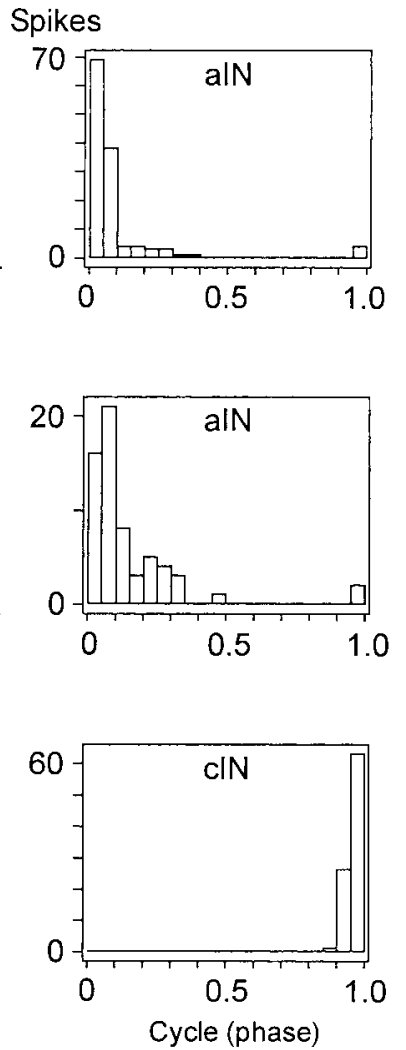

Figure 6. Firing patterns of aINs during swimming compared with those in premotor reciprocal inhibitory cINs. $A$, Example of aIN activity during swimming to show unreliability of firing and the possibility of two spikes per cycle. Dots indicate resting potential before swimming. $B$, Waterfall plot of a reliable aIN that fired on most swimming cycles. Each line shows a consecutive pair of cycles, normalized individually to a cycle phase of 1 . Cycles are defined by the start of consecutive ventral root ( $v r)$ bursts and corrected for the longitudinal spacing between aIN and vr recording positions (see Results for details). The second cycle on each line becomes the first cycle of the line below ( $n=20$ cycles). The histogram shows aIN spike timing in each cycle of swimming plotted for all spikes occurring in the first $6 \mathrm{sec}$ of a single swimming episode. $C$, Similar plots for a less reliable aIN with a broader distribution of firing times. $D$, Similar plots of the firing of a cIN showing very tightly distributed and reliable firing occurring just before the ventral root bursts.

responsible for the short-latency glycinergic IPSP seen in dlcs and rhythmically active spinal neurons after ipsilateral trunk skin stimulation (Roberts et al., 1985; Sillar and Roberts, 1992a,b; Zhao et al., 1998; Roberts, 2000). This IPSP has a latency of $\sim 10-15 \mathrm{msec}$ and inhibits EPSPs evoked at slightly shorter latencies by the same skin stimulus. The present sample of aINs was usually weakly excited by both trunk and head skin stimulation, but this excitation was variable from neuron to neuron (Fig. 11). When the ipsilateral trunk skin was stimulated $(n=16)$, EPSP

A

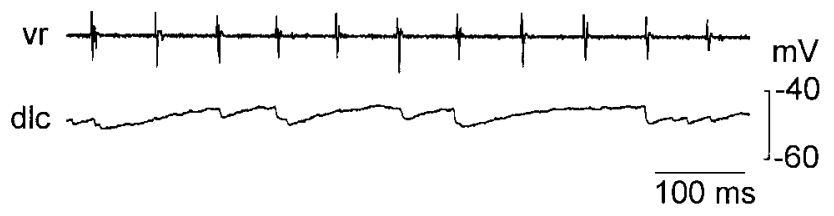

B

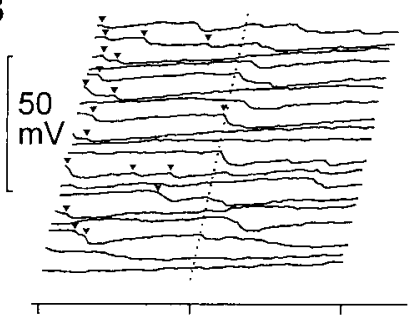

IPSPS

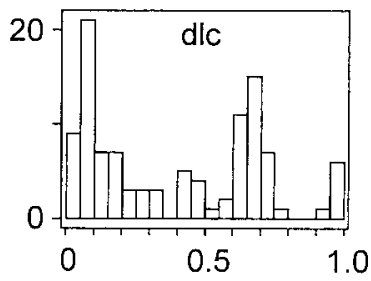

C
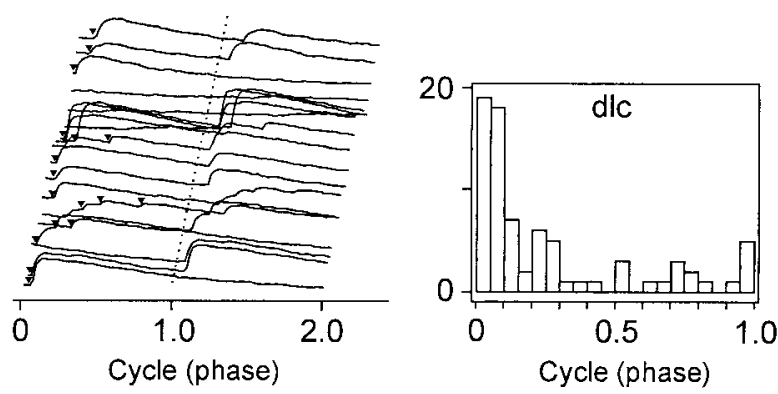

Figure 7. Timing of dlc IPSPs during swimming. A, Recording of dlc during swimming to show broadly on-cycle IPSPs in time with ipsilateral ventral root $(v r)$. $B$, Waterfall plot of dlc IPSPs during swimming (made clearer by depolarization; plotted as for Fig. 6) and histogram to show main on-cycle peak with another peak after mid-cycle. $C$, Another example in which IPSPs (reversed by hyperpolarization) occur mainly on-cycle. Onset of IPSPs is indicated (arrowheads).

latency was $6-16 \mathrm{msec}$ and amplitude was $2-16 \mathrm{mV}$. There is evidence from two paired recordings that cutaneous sensory Rohon-Beard neurons make direct excitatory synapses with some aINs, but these were not strong enough to produce an early aIN spike. After trunk skin stimulation, aIN excitation was never sufficient to evoke firing at a latency of $<20 \mathrm{msec}$. This makes it unlikely that members of the present sample of trunk aINs are responsible for the short-latency IPSP after trunk skin stimulation. When the head skin was stimulated, some EPSP latencies were shorter (range 4.7-31.3 msec; $n=15$ ). Although amplitudes were similar to those from trunk skin stimulation $(2-11 \mathrm{mV} ; n=$ 7), spikes were evoked at a short latency $(8.8$ and $9.8 \mathrm{msec})$ in two cases. This suggests that some aINs could produce short-latency ipsilateral inhibition in response to head skin stimulation.

When either head or trunk skin is stimulated repetitively at $20-30 \mathrm{~Hz}$ to imitate continuous local pressure, a slower pattern of motor activity is evoked, which we have called struggling (Soffe, 1991, 1993). In life, this vigorous alternating flexion movement can free the tadpole when attempts are made to grasp it. During repetitive skin stimulation, nearly all aINs (13 of 14 tested) fired bursts of spikes, whether or not they fired during swimming. When the ventral root record showed that struggling was evoked, 10 of 14 aINs tested fired bursts of spikes in phase with the 


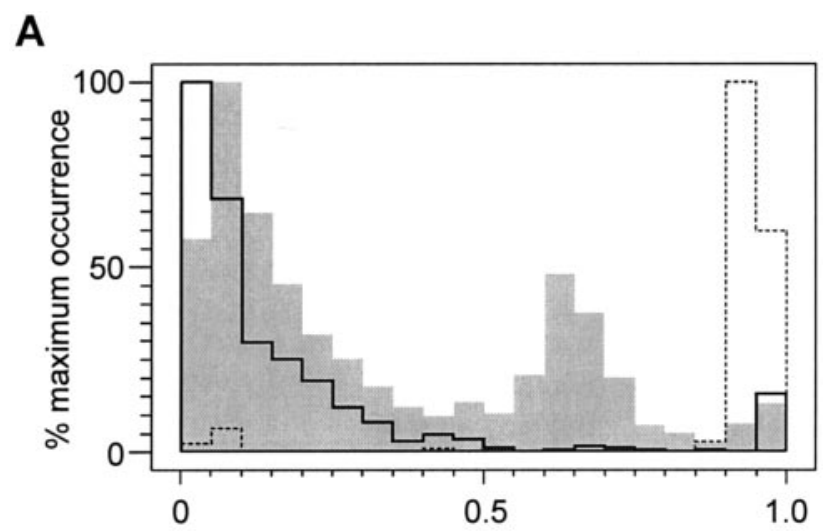

B

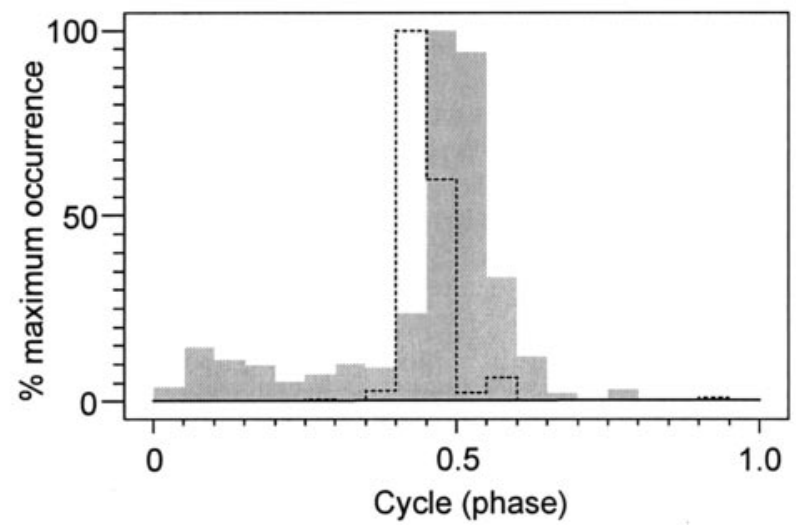

Figure 8. Phase histograms to show the relationship between the timing of interneuron spikes and IPSPs in dlcs and aINs during swimming. The cycle phase distributions for both spikes and IPSPs are normalized by plotting them as a percentage of their maximum occurrence. $A$, Spike timing in aINs (solid outline; $n=508$ spikes in 10 neurons in 10 animals) correlates closely with the main on-cycle peak of dlc IPSPs ( gray bars; $n=$ 849 IPSPs in 12 neurons in 8 animals). Spike timing in ipsilateral cINs (dashed outline; $n=293$ spikes in 5 neurons in 5 animals) does not relate to either of the two IPSP peaks. $B$, The mid-cycle peak of IPSPs in aINs (gray bars; $n=706$ IPSPs in 6 neurons in 6 animals) shows a good match to spike timing in cINs (dashed outline) once they have been phase advanced by 0.5 to model their contralateral counterparts.

ipsilateral ventral root. A simultaneous recording from aINs on both sides of the body shows this very clearly (Fig. 12).

\section{DISCUSSION}

Our new preparation, which permits whole-cell patch recording under visual control, has transformed the ease of making recordings from pairs of spinal neurons in Xenopus tadpoles. This method, used with neurobiotin filling, has allowed us to record from a large sample of aINs (Li et al., 2001) to examine their properties, synaptic connections, and activities during fictive behavior. We have shown that aINs are inhibitory and that they are active during swimming. We propose that by making direct synaptic connections with sensory pathway interneurons, they produce the main peak of phased glycinergic inhibition of these neurons, which has been shown to modulate responses to cutaneous stimulation during swimming (Sillar and Roberts, 1988, 1992a,b). The sample of aINs was recorded from the rostral half of the trunk spinal cord between the third and eighth postotic muscle segment (0.88-1.97 $\mathrm{mm}$ from midbrain). This sample had consistent properties, responses, and activities during fictive be-
A

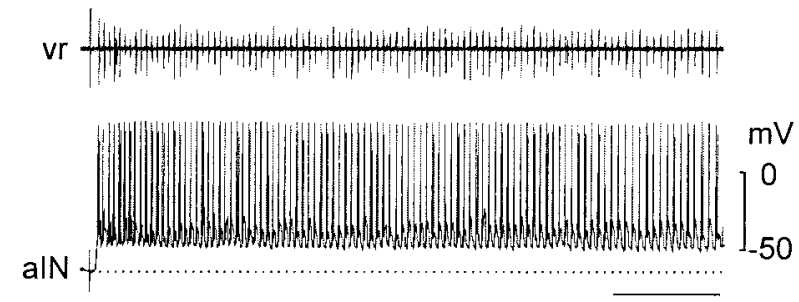

$1 \mathrm{~s}$
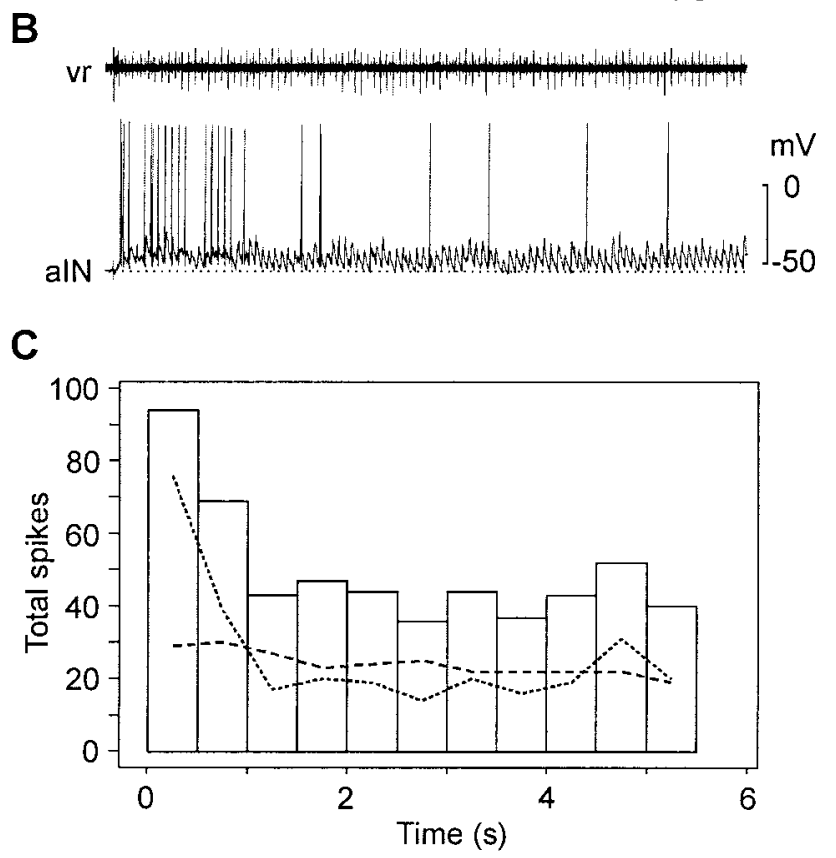

Figure 9. The dynamics of aIN firing during swimming episodes in two different experiments. $A, B$, aIN activity during the first $6 \mathrm{sec}$ of single swimming episodes. Nearly all aINs are active when swimming starts. A few continue firing throughout episodes $(A)$, but in most, firing becomes sporadic and generally declines with time $(B)$. C, Bar chart shows combined aIN spike numbers during the first $6 \mathrm{sec}$ of an episode $(n=17$ aINs). Less reliable aINs contribute to a peak of firing at the start of the episode (dotted line; $n=15$ ); reliable neurons, although a lower proportion, make a strong contribution subsequently (dashed line; $n=2$ ).

havior, which were distinct from those of other rhythmically active spinal interneurons and motoneurons studied previously (Dale, 1985, 1991; Soffe, 1990).

\section{Synaptic connections from alNs}

When aINs were recorded with sensory pathway dlcs, all synaptic connections from the aINs were inhibitory. A striking feature of these connections was the variability in IPSP amplitude and their unreliability. It is tempting to interpret this as evidence against the connections being monosynaptic. Certainly, the delays between presynaptic impulses in aINs and IPSPs in sensory pathway dlcs appear long when compared with mammal pathways at a higher temperature and using myelinated axons. However, the small synaptic delay $(\sim 1 \mathrm{msec}$ after subtracting conduction delays), the constant latency, and the observation of anatomical contacts make it highly implausible that the connections were not direct. Too few IPSPs were obtained for each recorded pair to allow detailed quantal analysis, but we suggest that the number of quanta released at each synaptic event is low (cf. Wall and Dale, 1993), and this may contribute to the frequent failures. 
A

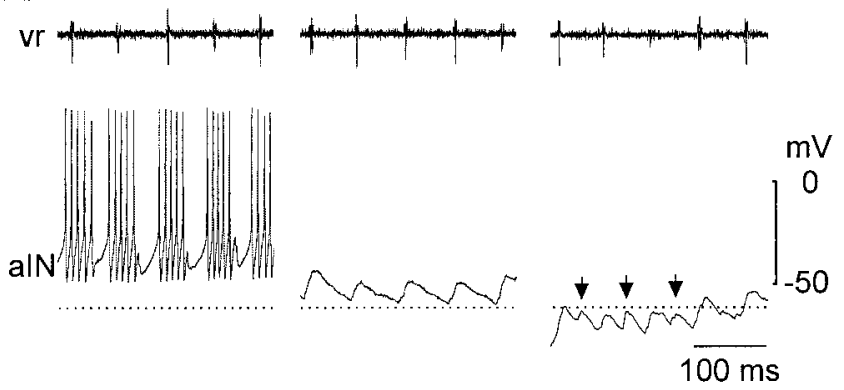

B
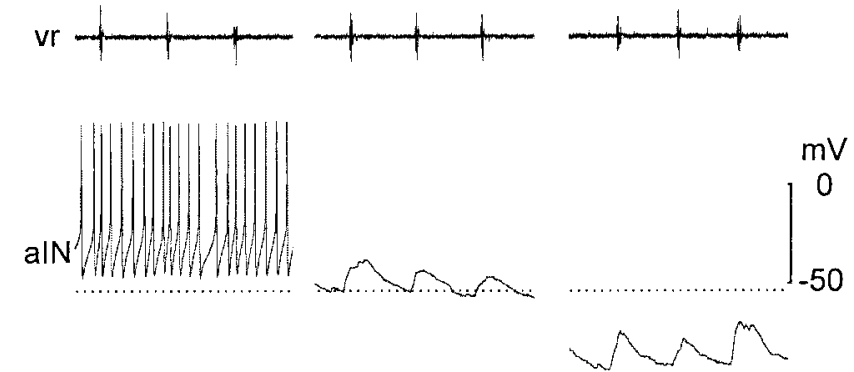

C

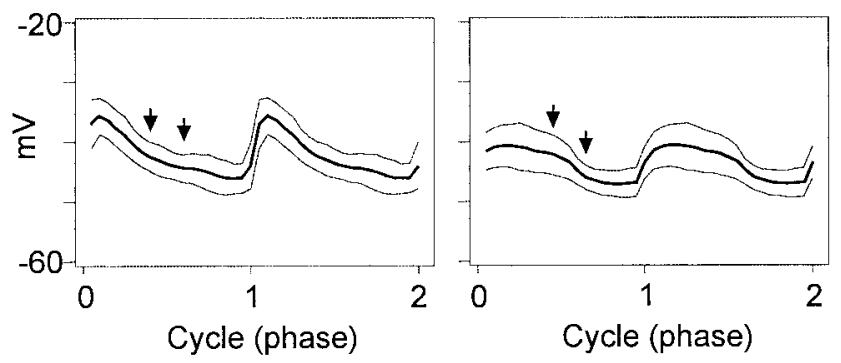

Figure 10. The pattern of synaptic drive to aINs during swimming shown by an ipsilateral ventral root record $(v r) . A, B$, Pattern of synaptic drive in two aINs revealed by injection of current; depolarizing is to the left, and hyperpolarizing is to the right. Dots, Resting potential. A, Depolarization leads to multiple firing with clear gaps indicative of mid-cycle inhibition, and hyperpolarization reveals reversed mid-cycle IPSPs (arrows). $B$, In this example, there is little evidence for mid-cycle inhibition because depolarization leads to continuous firing, and hyperpolarization does not reveal any IPSPs. $C, D$, Membrane potential trajectory during swimming in two additional aINs. Thick lines, Mean membrane potential trajectory over 20 cycles, calculated for 20 points per cycle; thin lines, SD. Arrows, Phases of 0.4 and 0.6. C, Clear peak in excitation and little sign of mid-cycle IPSP. $D$, Clear IPSP at mid-cycle and broader, rounded shape to excitation.

Because aINs have GABA-like immunoreactivity (Roberts et al., 1987; Li et al., 2001), it was surprising that the IPSPs that they produced were blocked by strychnine but not by bicuculline in all cases in which the pharmacology was examined. This indicates glycinergic transmission. Supporting this, the rise times of the IPSPs (2.2-6.4 msec) were similar to those for unitary glycinergic IPSPs recorded with sharp electrodes (Dale, 1985; Soffe et al., 2001), although the durations were longer (15.1-43.8 msec at $50 \%$ ), as might be expected during whole-cell recording when the input resistances and time constants of the neurons were larger. Reith and Sillar (1997) have suggested that GABA $_{\mathrm{A}}$ ergic IPSPs in this system are significantly longer than glycinergic IPSPs. Furthermore, the unitary conductances appeared similar to previous estimates for glycinergic synapses in the tadpole (1-1.7 nS)

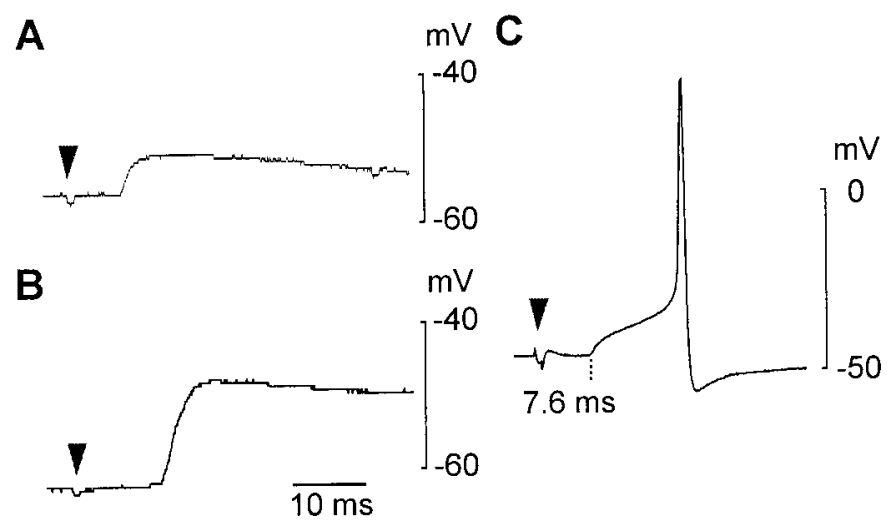

Figure 11. Responses of aINs to ipsilateral skin stimulation show that they are not excited to fire at short latency. $A-C$, Examples of responses to stimulation (arrowheads) to illustrate EPSPs evoked at shortest latencies seen and the spike with the shortest latency of $\sim 20 \mathrm{msec}(C)$ (EPSP latency indicated).

(Soffe et al., 2001). If the transmitter released by aINs is indeed glycine, why do they have GABA-like immunoreactivity (Roberts et al., 1987; Li et al., 2001)? One possible explanation might have been co-release (Jonas et al., 1998; O'Brien and Berger, 1999), but we have found no evidence for any GABA-mediated (bicuculline sensitive) component before or after strychnine application. It is also possible that aINs release GABA at an earlier stage of their development (Milner and Landmesser, 1999).

\section{Roles for alNs}

Because aINs produce inhibition of sensory pathway dlc interneurons, the first role that we have considered is inhibitory gating of the sensory pathway from skin touch to contalateral flexion response during swimming. Careful comparison of aIN spikes with dlc IPSPs during swimming allowed us to establish that there is a close correlation in their timing. Therefore, we propose that aINs are responsible for the large peak in dlc inhibition that starts broadly on-cycle in each swim cycle (Fig. 8). It is this peak in inhibition that defines the main cycle phase in which reflex contralateral flexion responses are gated out (Sillar and Roberts, 1988, 1992a). The earlier proposal that this peak of broadly on-cycle inhibition in dlcs came from the ipsilateral axons of some inhibitory cINs (Dale, 1985) now seems unlikely, because very few cINs that have been filled individually have such ipsilateral axons ( $<1 \%$; $n=455)$ (Yoshida et al., 1998; Li et al., 2001). In addition, we have now shown that the phase of discharge of cINs during swimming does not correlate with the IPSPs in dlcs; cIN spikes occur too soon in the cycle and are very tightly grouped compared with the IPSPs. The pattern of aIN firing during each swimming episode, with a peak in the first $\sim 1 \mathrm{sec}$ followed by a decline, mirrors inhibition in dlcs, which is also strong initially but becomes less reliable as episodes continue (Clarke and Roberts, 1984; Sillar and Roberts, 1988, 1992a; Soffe, 1993). Again, this contrasts with the firing pattern of cINs.

Broadly, on-cycle IPSPs are also seen in motoneurons and other ventrally located neurons that are active during swimming (Sillar and Roberts, 1993; Tunstall and Roberts, 1994). We have not analyzed these in detail but had been impressed previously by their unreliability and variable phasing. These features make it likely that these IPSPs are the result of aIN spikes. Unlike the on-cycle IPSPs in dlcs, the function of such IPSPs in rhythmically active neurons during swimming is not clear. Could they control 
A

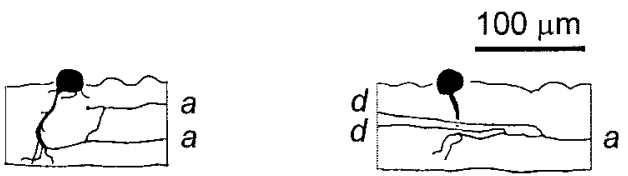

B

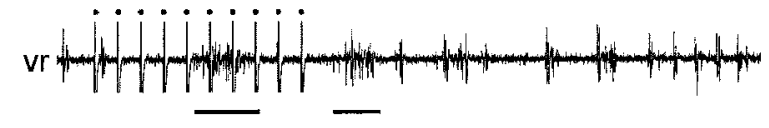

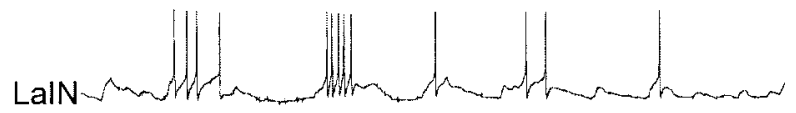

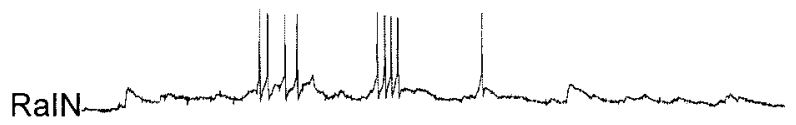

C
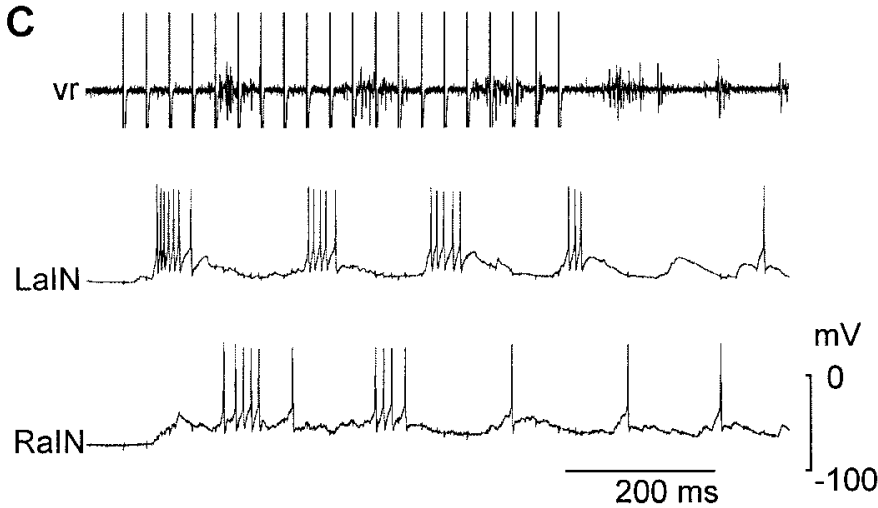

Figure 12. Activity of aINs during struggling evoked by repetitive skin stimulation. A, Scale diagrams in lateral view of two aINs recorded simultaneously. Shown are neuron from the left side (left) and neuron from the right side of the spinal cord (right), both at the fifth segment and viewed from the right side. $B, C$, Records to show alternating bursts of struggling activity in the two aINs shown in $A$ during repetitive stimulation of the skin on the tail $(B)$ and head $(C)$. The top trace in each record shows a recording of bursting struggling activity (above horizontal bars) in the ventral root on the right side $(v r)$, where repetitive stimuli are clear from artifacts (dots).

multiple firing, or are they the result of a lack of specificity in synapse formation in the young tadpole?

The phase-dependent modulation of skin sensory responses in immobilized tadpoles during swimming must originate in the swimming central pattern generator (CPG) (cf. Büschges and El Manira, 1998; Rudomin and Schmidt, 1999). However, although there is a ready source of rhythmic glycinergic inhibition in the spinal cord, both at mid-cycle from contralateral cINs and oncycle from ipsilateral cINs, this inhibition produced by the swimming CPG is apparently not used directly to modulate transmission of sensory information through the dlcs. Instead, CPG timing is modified by the use of a separate population of interneurons, the aINs, to provide rhythmic inhibition, which starts relatively late (just after the start of the cycle), is relatively prolonged (lasting well into each cycle), and shows a relatively strong occurrence at the very start of each episode compared with inhibition produced by cINs.

When the skin is stimulated repetitively and the ventral roots show the slow bursting activity typical of fictive struggling (Soffe,
1991), aINs fire vigorously (Fig. 12). This activity will produce the strong inhibitory gating of sensory pathway dlc interneurons described by Soffe (1993). If, as seems likely, aINs also inhibit ipsilateral rhythmically active neurons, then during struggling this inhibition will occur in phase with the activity of these neurons. It would alternate with reciprocal inhibition from the opposite side so the neurons would receive continuous inhibition. The function of on-cycle inhibition during struggling is also not clear. Could it help neurons to respond faster by reducing input resistance (time constant) and fire multiply by aiding in the repolarization process after an impulse?

When the tail skin is stimulated, the first contraction is normally on the opposite side of the body (Boothby and Roberts, 1995). One factor contributing to this asymmetry is a short latency ipsilateral IPSP (Roberts et al., 1985; Zhao et al., 1998). This is glycinergic, so it could come from aINs if they were excited to fire at short latency by skin stimulation. We have shown that this is not the case for the sample of aINs in this study. However, recordings from some more rostral aINs (data not shown) show slightly different responses. These interneurons receive only inhibition during swimming and so do not fire. However, they are active during struggling, and some of them are excited to fire at short latency after skin stimulation. They could therefore produce short-latency IPSPs on the same side as the stimulation. Their presence suggests that interneurons in a single anatomical class, with the same defining anatomical features, may not all have the same function but may separate into functional subclasses.

\section{Conclusions}

This study defines a class of spinal interneurons in the hatchling Xenopus tadpole that are responsible for inhibitory gating of sensory pathway interneurons during swimming. Ascending interneurons have unipolar somata, mainly ventral dendrites, and an ascending ipsilateral axon forming a long ipsilateral descending branch (Li et al., 2001). Anatomically homologous interneurons are present in the spinal cord of the young newt tadpole (Triturus vulgaris) (Harper and Roberts, 1993) and the zebrafish embryo, where they are called circumferential ascending (Bernhardt et al., 1990; Roberts, 2000). We predict that all of these interneurons will have a similar function in producing phased inhibition of sensory pathway interneurons during swimming. Our hope is that the functional and anatomical definition of ascending interneurons will soon be followed by a definition of the transcriptional regulation of their identity (Lee and Pfaff, 2001). To evaluate the broader significance of our results, the later development of aINs and their relation to inhibitory interneurons with related roles in the adult spinal cord need to be established (Baev, 1980; Baev and Kostyuk, 1982; Gossard and Rossignol, 1990; Rudomin et al., 1998).

\section{REFERENCES}

Alford S, Christensen J, Grillner S (1991) Presynaptic GABA ${ }_{A}$ and $\mathrm{GABA}_{\mathrm{B}}$ receptor-mediated phasic modulation in axons of spinal motor interneurons. Eur J Neurosci 3:107-117.

Baev KV (1980) Polarization of primary afferent terminals in the lumbar spinal cord during fictitious locomotion. Neurophysiology 12:305-311.

Baev KV, Kostyuk PG (1982) Polarization of primary afferent terminals of lumbosacral cord elicited by the activity of spinal locomotor generator. Neuroscience 7:1401-1409.

Bernhardt RR, Chitnis AB, Lindamer L, Kuwada JY (1990) Identification of spinal neurons in the embryonic and larval zebrafish. J Comp Neurol 302:603-616.

Boothby K, Roberts A (1995) Effects of site of tactile stimulation on the 
escape swimming responses of hatchling Xenopus laevis embryos. J Zool Lond 235:113-125.

Buchanan JT (2001) Contributions of identifiable neurons and neuron classes to lamprey vertebrate neurobiology. Prog Neurobiol 63:441-446.

Büschges A, El Manira A (1998) Sensory pathways and their modulation in the control of locomotion. Curr Opin Neurobiol 8:733-739.

Clarke JDW, Roberts A (1984) Interneurones in the Xenopus embryo spinal cord: sensory excitation and activity during swimming. J Physiol (Lond) 354:345-362.

Clarke JDW, Hayes BP, Hunt SP, Roberts A (1984) Sensory physiology, anatomy and immunohistochemistry of Rohon-Beard neurones in embryos of Xenopus laevis. J Physiol (Lond) 348:511-525.

Dale N (1985) Reciprocal inhibitory interneurons in the Xenopus embryo spinal cord. J Physiol (Lond) 363:61-70.

Dale N (1991) The isolation and identification of spinal neurons that control movement in the Xenopus embryo. Eur J Neurosci 3:1025-1035.

Dale N, Roberts A, Soffe SR (1990) The anatomy, development, physiology and role of glycinergic neurons in the Xenopus embryo spinal cord. In: Glycine neurotransmission (Ottersen O-P, Storm-Mathisen J, eds), pp 329-353. Chichester: Wiley.

El Manira A, Tegner J, Grillner S (1996) Locomotor-related presynaptic modulation of primary afferents in the lamprey. Eur $\mathrm{J}$ Neurosci 9:696-705

Gossard JP, Rossignol S (1990) Phase-dependent modulation of dorsal root potentials evoked by peripheral nerve stimulation during fictive locomotion in the cat. Brain Res 537:1-13.

Harper CE, Roberts A (1993) Spinal cord neuron classes in embryos of the smooth newt Triturus vulgaris: a horseradish peroxidase and immunocytochemical study. Philos Trans R Soc Lond B Biol Sci 340:141-160.

Jonas P, Bischofberger J, Sandkuhler J (1998) Corelease of two fast neurotransmitters at a central synapse. Science 281:419-424.

Kirk MD, Wine JJ (1984) Identified interneurons produce both primary afferent depolarization and presynaptic inhibition. Science 225:854-856.

Lee S-K, Pfaff SL (2001) Transcriptional networks regulating neuronal identity in the developing spinal cord. Nat Neurosci 4:1183-1191.

Li W-C, Perrins R, Soffe SR, Yoshida M, Walford A, Roberts A (2001) Discriminating classes of spinal interneuron in Xenopus tadpoles. J Comp Neurol 441:248-265.

Milner LD, Landmesser LT (1999) Cholinergic and GABAergic inputs drive patterned spontaneous motoneuron activity before target contact. J Neurosci 19:3007-3022.

Nieuwkoop PD, Faber J (1956) Normal tables of Xenopus laevis (Daudin). Amsterdam: North-Holland.

O'Brien JA, Berger AJ (1999) Cotransmission of GABA and glycine to brainstem motoneurons. J Neurophysiol 82:1638-1641.

Pearson KG (1993) Common principles of motor control in vertebrates and invertebrates. Annu Rev Neurosci 16:265-297.

Reith CA, Sillar KT (1997) Pre- and postsynaptic modulation of spinal GABAergic neurotransmission by the neurosteroid, $5 \beta$-prengan- $3 \alpha$-ol20-one. Brain Res 770:202-212.

Roberts A (2000) Early functional organisation of spinal neurons in developing lower vertebrates. Brain Res Bull 53:585-593.

Roberts A, Clarke JDW (1982) The neuroanatomy of an amphibian embryo spinal cord. Philos Trans R Soc Lond B Biol Sci 296:195-212.

Roberts A, Sillar KT (1990) Characterisation and function of spinal excitatory interneurons with commissural projections in Xenopus laevis embryos. Eur J Neurosci 2:1051-1062.

Roberts A, Dale N, Evoy WH, Soffe SR (1985) Synaptic potentials in motoneurons during fictive swimming in spinal Xenopus embryos. J Neurophysiol 54:1-10.

Roberts A, Dale N, Ottersen OP, Storm-Mathisen J (1987) The early development of neurons with GABA immunoreactivity in the central nervous system of Xenopus laevis embryos. J Comp Neurol 261:435-449.

Roberts A, Soffe SR, Perrins R (1997) Spinal networks controlling swimming in hatchling Xenopus tadpoles. In: Neurons, networks and motor behavior (Stein PSG, Grillner S, Selverston AI, Stuart DG, eds), pp 83-89. Boston: MIT.

Roberts A, Soffe SR, Wolf ES, Yoshida M, Zhao F-Y (1998) Central circuits controlling locomotion in young frog tadpoles. Ann NY Acad Sci 860:19-34.

Rossignol S, Lund JP, Drew T (1988) The role of sensory inputs in regulating the pattern of rhythmical movements in higher vertebrates. In: Neural control of rhythmic movements in vertebrates (Cohen A, Rossignol S, Grillner S, eds), pp 201-283. New York: Wiley.

Rudomin P, Schmidt RF (1999) Presynaptic inhibition in the vertebrate spinal cord revisited. Exp Brain Res 129:1-37.

Rudomin P, Romo R, Mendell L (1998) Presynaptic inhibition and neural control. Oxford: Oxford UP.

Sillar KT, Roberts A (1988) A neuronal mechanism for sensory gating during locomotion in a vertebrate. Nature 331:262-265.

Sillar KT, Roberts A (1992a) Phase-dependent modulation of a cutaneous sensory pathway by glycinergic inhibition from the locomotor rhythm generator in Xenopus embryos. Eur J Neurosci 4:1022-1034.

Sillar KT, Roberts A (1992b) The role of premotor interneurones in phase-dependent modulation of a cutaneous reflex during swimming in Xenopus laevis embryos. J Neurosci 12:1647-1657.

Sillar KT, Roberts A (1993) Control of frequency during swimming in Xenopus embryos: a study on interneuronal recruitment in a spinal rhythm generator. J Physiol (Lond) 472:557-572.

Soffe SR (1990) Active and passive membrane properties of spinal cord neurons which are rhythmically active during swimming in Xenopus embryos. Eur J Neurosci 2:1-10.

Soffe SR (1991) Triggering and gating of motor responses by sensory stimulation: behavioural selection in Xenopus embryos. Proc R Soc Lond B Biol Sci 246:197-203.

Soffe SR (1993) Two distinct rhythmic motor patterns are driven by common premotor and motor neurons in a simple vertebrate spinal cord. J Neurosci 13:4456-4469.

Soffe SR, Roberts A (1982) Tonic and phasic synaptic inputs to spinal cord motoneurones active during fictive locomotion in frog embryos. J Neurophysiol 48:1279-1288.

Soffe SR, Clarke JDW, Roberts A (1984) Activity of commissural interneurones in spinal cord of Xenopus embryos. J Neurophysiol 51:1257-1267.

Soffe SR, Zhao F-Y, Roberts A (2001) Functional projection distances of spinal interneurons mediating reciprocal inhibition during swimming in Xenopus tadpoles. Eur J Neurosci 13:617-627.

Tunstall MJ, Roberts A (1991) Longitudinal coordination of motor output during swimming in Xenopus embryos. Proc R Soc Lond B Biol Sci 244:27-32.

Tunstall MJ, Roberts A (1994) A longitudinal gradient of synaptic drive in the spinal cord of Xenopus embryos and its role in coordination of swimming. J Physiol (Lond) 474:393-405.

Wall MJ, Dale N (1993) GABA $_{\mathrm{B}}$ receptors modulate glycinergic inhibition and spike threshold in Xenopus embryo spinal neurones. J Physiol (Lond) 469:275-290.

Yoshida M, Roberts A, Soffe SR (1998) Axon projections of reciprocal inhibitory interneurons in the spinal cord of young Xenopus tadpoles and implications for the pattern of inhibition during swimming and struggling. J Comp Neurol 400:504-518.

Zehr EH, Stein RB (1999) What functions do reflexes serve during human locomotion? Prog Neurobiol 58:185-205.

Zhao F-Y, Burton BG, Wolf ES, Roberts A (1998) Asymmetries in sensory pathways from skin to motorneurons on each side of the body determine the direction of an avoidance response in hatchling Xenopus tadpoles. J Physiol (Lond) 506:471-487. 